\title{
Generation and Analyses of Guided Waves in Planar Structures
}

\author{
Enkelejda Sotja ${ }^{1}$, P. Malkaj ${ }^{2}$, Dhimiter Sotja ${ }^{2}$ \\ ${ }^{1}$ Department of Manufacturer-Management, Polytechnic University of Tirana, Tirana, Albania \\ ${ }^{2}$ Department of Mechanics, Polytechnic University of Tirana, Tirana, Albania \\ E-mail:esotja@yahoo.com,sotja@icc-al.org \\ Received February 11, 2011; revised March 22, 2011; accepted April 8, 2011
}

\begin{abstract}
Guided wave in plate propagates like shear waves and Lamb waves. Both kinds are very dispersive waves. Generation and analysis of dispersion curves is very important. Those are used to predict and describe the relation between frequency, thickness with phase velocity, group velocity and wave mode. For a stainless steel plate with thickness $5.89 \mathrm{~mm}$ we built dispersion curves for shear and Lamb waves. A method based on peak frequency shifts of the shear waves along with the thickness was applied. In line with dispersion curves of shear waves phase velocity was seen that mode of waves translate in some points, have experiment performance much better than other points.
\end{abstract}

Keywords: Guided Wave, Dispersion Curves, Shear Wave, Lamb Wave

\section{Introduction}

Low frequency ultrasonic waves propagate in long distance in the league (meters compared to millimeters in conventional techniques in UT), with small loss of energy, which are called "directed waves" (guided wave).

Guided waves monitor large areas from a single position even when the objects are not fully physically accessible, and they propagate standing localized between surfaces of a thin-walled structure, and even it is curved. These properties make them important for the ultrasonic control of facilities of special importance as airplanes, helicopters, spaceship, pressure vessels, and oil deposits. A very important use of guided waves is in the control of oil and gas pipelines and heat city systems regardless of their underground or underwater location [1].

Guided waves are cited as elastic waves that propagate in the samples where the axial dimension is many times greater than the dimensions of the section. The energy of these waves is guided from the boundaries of the sample with the environment, or other materials [2].

For each object's form and for any combination of dimensions (e.g. section size or the internal and external diameter), there is now a unique set of wave dissemination or the wave's mode. Any one of these wave's mode will be propagate with a set pattern, known as the shape of the mode [3].

The changing of the wave mode frequency is associ- ated with the change of shape of the mode, phase's velocity, group's velocity. In order to predict the relation between frequency, the thickness of the structure and other parameters mentioned above there are used dispersion curves (so called because the change of the frequency brings the change of wave velocity and vibrations tent to disperse during the spread). The data in these curves are used to do the tests controlling the structures, ranging from defining the generating equipment parameters of the guided waves in the structures until the final calculations [4].

\section{Overview of the Propagation of the Guided Waves in the Plan Structures}

The simple forms of ultrasonic guided waves in plan structures are shear (transversal) waves. Movement of particles to the shear wave polarized parallel to the surface of the flat structure and perpendicular to the direction of the spread of the wave. Shear waves appear symmetrical or asymmetrical. All modes are dispersive with exception of the base mode of the spread.

Lamb waves are guided waves the most complicated ones that propagate in a structure. Lamb waves propagate according to two basic wave mode, symmetric Lamb wave $S_{0}, S_{1}, S_{2}$, and non-symmetric Lamb wave $A_{0}, A_{1}$, $A_{2}$, both are dispersive types. Greater value of $f \cdot d$ causes bigger number of the Lamb wave's modes that exist si- 
multaneously. For small values of the product $f \cdot d$ may exist only the basic mode Lamb wave (symmetrical and asymmetrical $\mathrm{S}_{0}, \mathrm{~A}_{0}$ ) [5].

\section{Spread of the Guided Wave Run in Plate Structures (Mathematical Formalism)}

In an elastic isotropic environment, waves propagate freely in all directions. Using the 3D elasticity theory in which the vector equation of the movement according Navier's equations is [6]:

$$
(\lambda+\mu) \boldsymbol{\nabla}(\boldsymbol{\nabla} \cdot \boldsymbol{u})+\mu \nabla^{2} \boldsymbol{u}=\rho \ddot{\boldsymbol{u}}
$$

$\lambda$ and $\mu$ are Lame constants, $\rho$ is mass density, and $u$ is the displacement vector. Displacement vector in solid bodies is given in function of the two potential functions, a scalar potential $\Phi$ and a vector potential

$\boldsymbol{H}=H_{x} \boldsymbol{i}+H_{y} \boldsymbol{j}+H_{z} \boldsymbol{k}$ which is known as Helmholtz' solution,

$$
\boldsymbol{u}=\boldsymbol{\nabla} \Phi+\boldsymbol{\nabla} \times \boldsymbol{H}
$$

Rewrite the vector equation of movement by using the Equation (2), it is benefited the equation of the wave for potential scalar function $\Phi$ and vector potential $\boldsymbol{H}$.

$$
\begin{aligned}
c_{p}^{2} \nabla^{2} \Phi & =\nabla \ddot{\Phi} \\
c_{s}^{2} \nabla^{2} \vec{H} & =\ddot{\boldsymbol{H}}
\end{aligned}
$$

where the potential scalar propagates with $c_{p}$, longitudinal wave speed, and vector potential propagate with $c_{s}$, shear wave speed.

Recognize 3-D waves with straight crested. In straightcrested waves, the wave front is parallel to the axis $\mathrm{z}$ and the wave spread along $\mathrm{z}$ does not change (Figure 1). All the functions included in the analysis do not depend on $\mathrm{z}$ and the normal wave front will be perpendicular to the axis $z, \boldsymbol{n} \perp \boldsymbol{k}$ where $\boldsymbol{k}$ is the unit vector of $\mathrm{z}$ axis so,

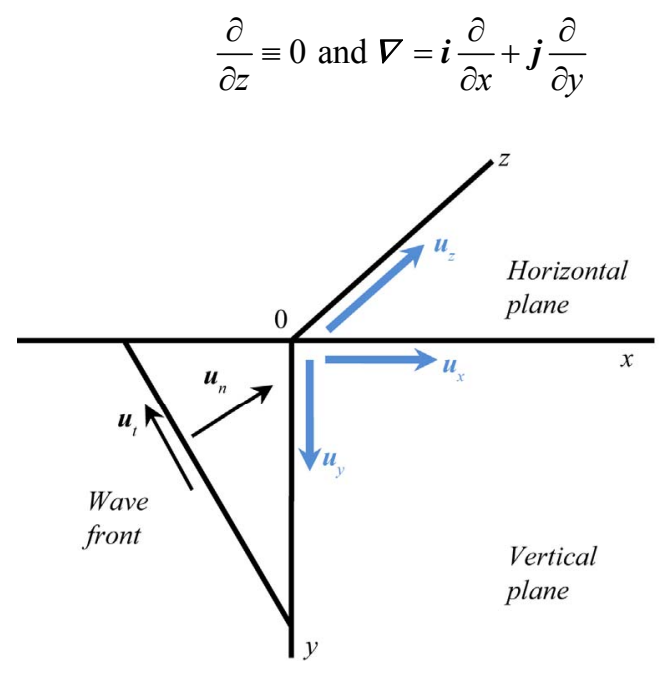

Figure 1. z-invariant plane of 3Dwaves.
Replacement of Equation (4) to (2) shows that displacement has components in all three directions $(x, y, z)$,

$$
\boldsymbol{u}=\boldsymbol{i}\left(\frac{\partial \phi}{\partial x}+\frac{\partial H_{Z}}{\partial y}\right)+\boldsymbol{j}\left(\frac{\partial \phi}{\partial y}+\frac{\partial H_{Z}}{\partial x}\right)+\boldsymbol{k}\left(\frac{\partial H_{Y}}{\partial x}+\frac{\partial H_{X}}{\partial y}\right)(5)
$$

The equation of wave for potentials scalar function $\Phi$, $H_{x}, H_{y}, H_{z}$ which satisfy the above conditions become,

$$
c_{p}^{2} \nabla^{2} \Phi=\ddot{\Phi}\left\{\begin{array}{l}
c_{S}^{2} \nabla^{2} H_{x}=\ddot{H}_{x} \\
c_{S}^{2} \nabla^{2} H_{y}=\ddot{H}_{y} \\
c_{S}^{2} \nabla^{2} H_{z}=\ddot{H}_{z}
\end{array} \quad \frac{\partial H_{x}}{\partial x}+\frac{\partial H_{y}}{\partial y}+\frac{\partial H_{z}}{\partial z}=0\right.
$$

For a plate sample with upper and lower surface without tension, set $\mathrm{y}= \pm \mathrm{d}$ (Figure 2), lied to infinity in directions $\mathrm{x}$ and $\mathrm{z}$, government equations which satisfy the scalar and vector potential functions of the waves are,

$$
\nabla^{2} \Phi=\frac{1}{c_{p}^{2}} \frac{\partial^{2} \Phi}{\partial \tau^{2}}, \nabla^{2} \boldsymbol{H}=\frac{1}{c_{s}^{2}} \frac{\partial^{2} \boldsymbol{H}}{\partial \tau^{2}} \text { dhe } \boldsymbol{\nabla} \cdot \boldsymbol{H}=0
$$

Replacement in these governing equations the scalar and vector functions of the wave (6) leads us in the form of Equation (8),

$$
\begin{aligned}
& f^{\prime \prime}-\xi^{2} f=-\omega^{2} f / c_{p}^{2} \\
& h_{x}^{\prime \prime}-\xi^{2} h_{x}=-\omega^{2} h_{x} / c_{s}^{2} \\
& h_{y}^{\prime \prime}-\xi^{2} h_{y}=-\omega^{2} h_{y} / c_{s}^{2} \\
& h_{z}^{\prime \prime}-\xi^{2} h_{z}=-\omega^{2} h_{z} / c_{s}^{2}
\end{aligned}
$$

where $\xi$ is wavenumber, $\omega$ is circular frequency, $c_{p}^{2}=(\lambda+2 \mu) / \rho$ and $c_{s}^{2}=\mu / \rho$ are the longitudinal wave velocity and shear wave velocity. Solution of Equation (8) states,

$$
\begin{aligned}
& \Phi=(M \cos \alpha y+N \sin \alpha y) e^{i(\xi x-\omega \tau)} \\
& H_{x}=(K \cos \beta y+L \sin \beta y) e^{i(\xi x-\omega \tau)} \\
& H_{y}=(T \cos \beta y+P \sin \beta y) e^{i(\xi x-\omega \tau)} \\
& H_{z}=(R \cos \beta y+V \sin \beta y) e^{i(\xi x-\omega \tau)}
\end{aligned}
$$

where $\alpha^{2}=\left(\frac{\omega^{2}}{c_{p}^{2}}\right)-\xi^{2}$, and $\beta^{2}=\left(\frac{\omega^{2}}{c_{s}^{2}}\right)-\xi^{2}$. Constants $\mathrm{M}$ till $\mathrm{V}$ determined by considering free surface from constraints, which leads us to the Equation (10) with,

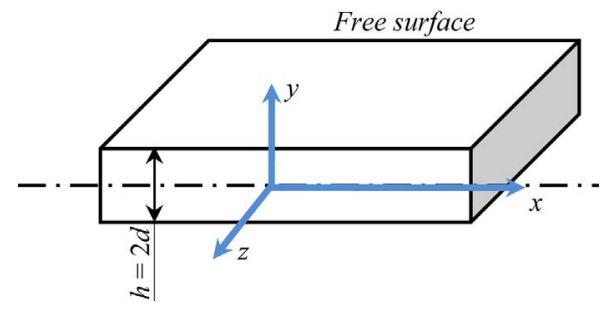

Figure 2. Flat sample with $2 d$ thickness, lying in the infinite in $x$ and $z$ directions. 
$c_{1}=(\lambda+2 \mu) \alpha^{2}+\lambda \xi^{2}$ and $c_{2}=2 i \mu \xi \beta$,

$c_{3}=2 i \xi \alpha, c_{4}=\xi^{2}-\beta^{2}, c_{5}=i \xi \beta$.

(See below (10))

Analyzing (10) shows that both types of shear and Lamb waves can be generated from the same set of Equations. The first two couples correspond to symmetric and asymmetric Lamb waves, and last two couples correspond to symmetric and asymmetric shear waves.

So, in a plate sample with upper and lower surface without tension set at $y= \pm d$, extended to infinite in $x$ and $z$ directions, guided ultrasonic waves propagate as the Lamb wave and shear wave. Lamb waves spread vertically polarized and shear wave spread horizontally polarized. Both types of waves are composed of symmetrical or asymmetrical waves tide against plan that passes in center of the planar sample.

\section{Relation of. Wave Speed versus Frequency Thickness (Dispersion Curves)}

In addition to finding a nontrivial solution, homogeneous Equation (9) should have the determinant of the set of the matrix coefficients equal to zero. The determinant can be expressed as the product of four smaller determinants which correspond to couple coefficients $(\mathrm{M}, \mathrm{V}),(\mathrm{N}$, $\mathrm{R})$, (T, L) (K, P).

These equations are solved numerically allowing the determination of the possible guided waves. Any solution of the characteristic equation, defines a specific value of the wave number $\xi$, and so the wave velocity c. Find of the solutions leads to the relation of phase velocity versus frequency or frequency thickness. These values are presented in the form of dispersion curve [6].

For Lamb waves there are constructed dispersion curves of the phase velocity (Figure 3). For low values of the frequency-thickness product, there are only two types of Lamb waves: $\mathrm{S}_{0}$, which is symmetric Lamb wave and it is similar to longitudinal waves, and $\mathrm{A}_{0}$, which is an asymmetric Lamb wave and it is similar to bending waves.

For high values of the product of frequency multiplied

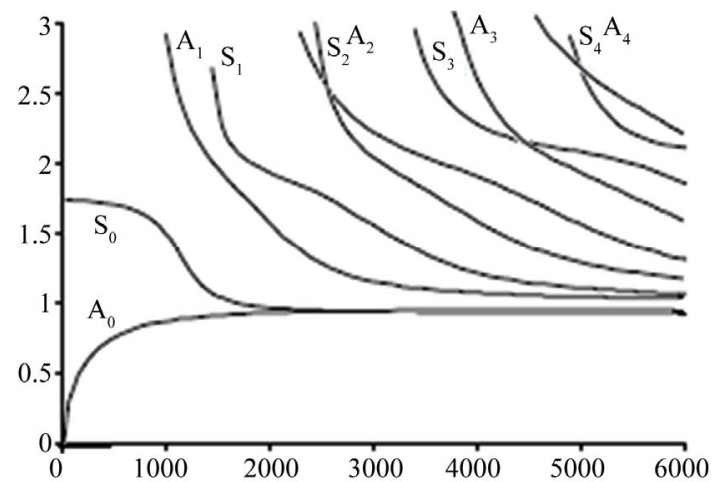

Figure 3. Dispersion curves of the phase velocity in plate structure; $S_{n}$ symmetric Lamb waves; $A_{n}$ asymmetric Lamb waves.

to thickness, there are present Lamb waves, $S_{n}$ and $A_{n}$, where $\mathrm{n}=0,1,2, \cdots, \mathrm{n}$ etc. For high frequencies, and $\mathrm{A}_{0}$, $\mathrm{S}_{0}$ Lamb waves become Rayleigh surface waves, which lie on the upper and lower surfaces of plate structure.

Lamb waves are highly dispersive (phase velocity varies significantly with the change of frequency). However, $\mathrm{S}_{0}$ wave for low values of the frequency- thickness product shows small dispersion.

\section{Techniques of the Guided Wave Generation at the Dispersion Curves}

There are used two techniques for the generation of guided waves the first one is with the angular probe where the energy within the structure can be calculated through Snellit's law. Every angle of probe defines one horizontal activation line in dispersion curves graphs (Figure 4, dot horizontal lines). By changing the frequency becomes possible the activation of the wave's modes along these horizontal lines.

The second technique is realized with a comb probe where the spaces between the elements determine the slope of activation line graphs of the dispersion curve. The line is shown for a particular space, under which, by changing the frequency move along its beginning at the origin (Figure 4, continuous slope line, $\lambda=\mathrm{V} / \mathrm{f}$ ). It is

$$
\left[\begin{array}{cccccccc}
-c_{3} \sin \alpha d c_{4} \sin \beta d & 0 & 0 & 0 & 0 & 0 & 0 \\
c_{1} \cos \alpha d c_{2} \cos \beta d & 0 & 0 & 0 & 0 & 0 & 0 \\
0 & 0 & c_{1} \sin \alpha d-c_{2} \sin \beta d & 0 & 0 & 0 & 0 \\
0 & 0 & c_{3} \cos \alpha d c_{4} \cos \alpha d & 0 & 0 & 0 & 0 \\
0 & 0 & 0 & 0 & -c_{5} \sin \beta d \beta^{2} \sin \beta d & 0 & 0 \\
0 & 0 & 0 & 0 & -\beta \sin \beta d i \xi \sin \beta d & 0 & 0 \\
0 & 0 & 0 & 0 & 0 & 0 & \beta^{2} \cos \beta d c_{5} \sin \beta d \\
0 & 0 & 0 & 0 & 0 & 0 & i \xi \cos \beta d \beta \cos \beta d
\end{array}\right] \cdot\left(\begin{array}{l}
M \\
V \\
N \\
R \\
T \\
L \\
K \\
P
\end{array}\right)=0
$$


possible to change the slope of line through change of the space, or by using some delay elements in the probe comb. In both techniques, maximum amplitude can be reached where the activation lines suspend the mode of the waves [7].

\section{Direct Method of Testing a Planar Structure with Guided Waves}

Test consists on thickness control of a reference plate with known thickness $5.89 \mathrm{~mm}$, material, by using direct method [8]. The transducer shear horizontal Emat [9] (electromagnetic transducer) with wavelengths' $\lambda=12$ $\mathrm{mm}$, the ultrasonic device Epoch $4 \mathrm{~b}$. There are generated the dispersion curves for Lamb waves and shear waves for the stainless steel plate which are illustrated in Figure 5 for phase velocity and group velocity versus frequency-thickness product. Dispersion curves for Lamb waves are shown in Figure 6.

Length of wave to shear wave is fixed through Emat magnet probe, which produces an activation line to the dispersion curves of the phase velocity versus frequency thickness product, slope line is shown to Figure $\mathbf{5}(\mathbf{b})$. The slope of activation line is determined by wavelength of the transducer.

When the activation line permeates the dispersion curve of the phase of velocity will be noted a maximum in amplitude. The corresponding frequency is called "peak frequency".

A tone-burst function generator that performs a frequency sweep was used to do the thickness measurement. The amplitudes of the received signals were recorded as the frequencies were swept from low to high. Figure 7 shows the results of frequency sweeping.

Mode $\mathrm{n} 0$ is not dispersive and independent of the thickness changes, so peak frequency in n0 mode of the

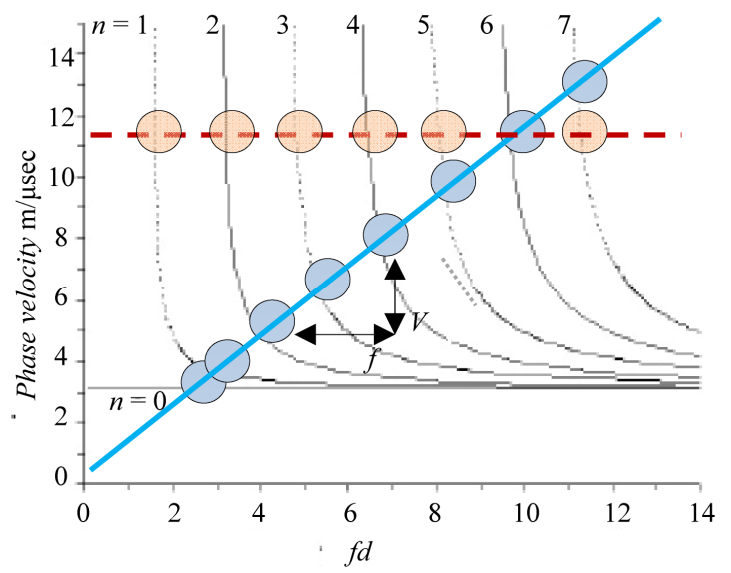

Figure 4. Dispersion curves and the activation lines of the guided waves.

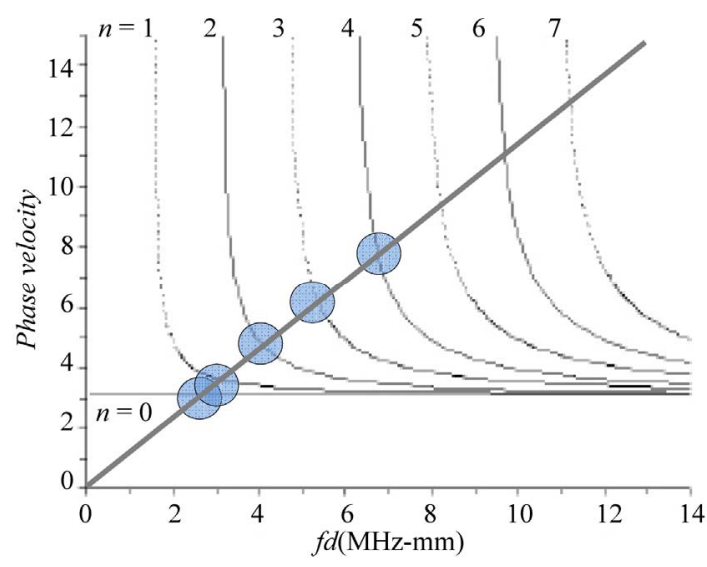

(a)

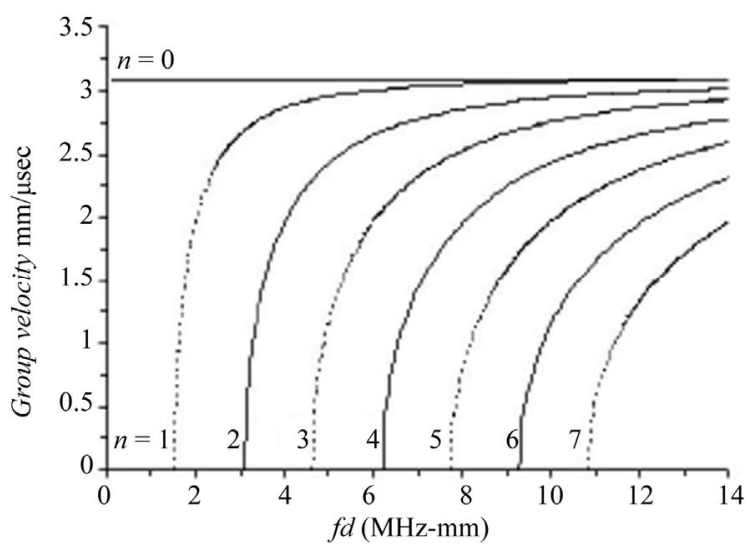

(b)

Figure 5. Shear waves dispersion curves for stainless steel plate with thickness $5.89 \mathrm{~mm}$. (a) phase velocity $(\mathrm{mm} / \mu \mathrm{sec})$; (b) group velocity ( $\mathrm{mm} / \mu \mathrm{sec})$.

wave is non-changeable for the entire structure, this quality makes this wave mode unsuitable to measure the thickness. 0 peak frequency is determined by the wavelength for the Emat transducer and phase velocity, which is also the shear wave velocity in the material and it, is determined by the properties of the material.

This has value because it can be used to see clearly to what kind of wave belongs a peak frequency.

As example, in an experiment by changing the frequency can not be predict the peak frequency value for $\mathrm{nl}$, $\mathrm{n} 2$ or other types, but we are able to understand the wave mode by comparing peak frequency with fixed frequency of n0 (Figure 7).

If this peak frequency is the first one observed after $\mathrm{n} 0$, it should belong to $\mathrm{nl}$ mode of the wave; if is the second peak frequency it belongs to $\mathrm{n} 2$ mode wave and so on. By recognizing the value of peak frequency and wavelength, the phase velocity can be calculated as product of peak frequency with wavelength.

Then the values of the product fd (frequency-thickness) 


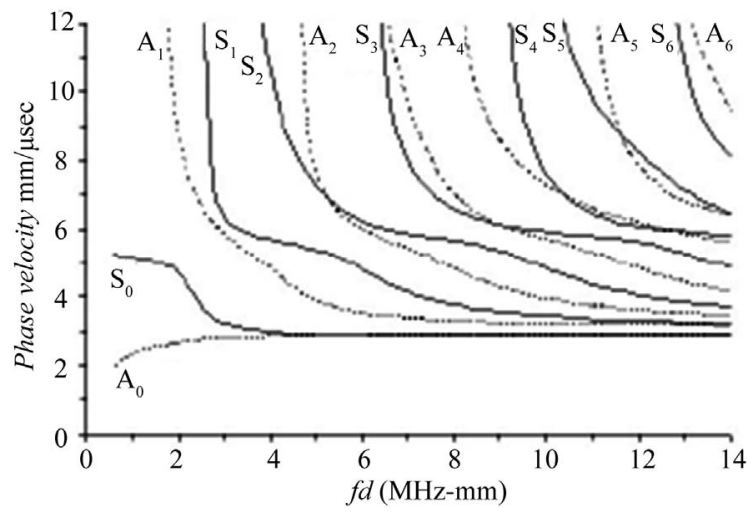

(a)

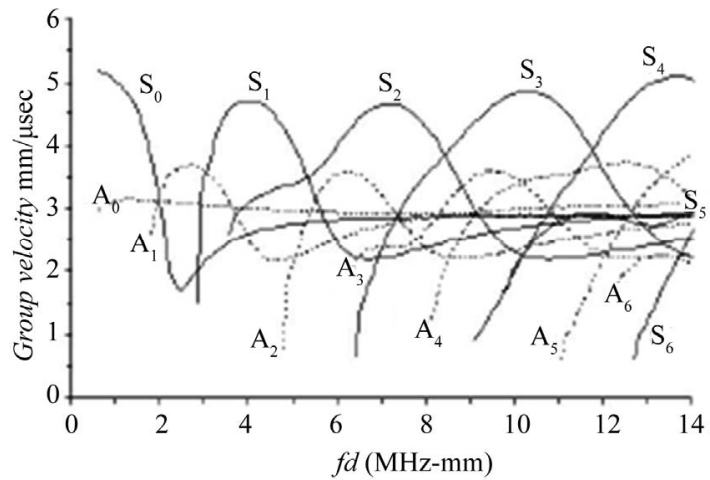

(b)

Figure 6. Lamb waves dispersion curves for stainless steel plate with thickness $5.89 \mathrm{~mm}$. (a) phase velocity $(\mathrm{mm} / \mu \mathrm{sec})$; (b) group velocity $(\mathrm{mm} / \mu \mathrm{sec})$.

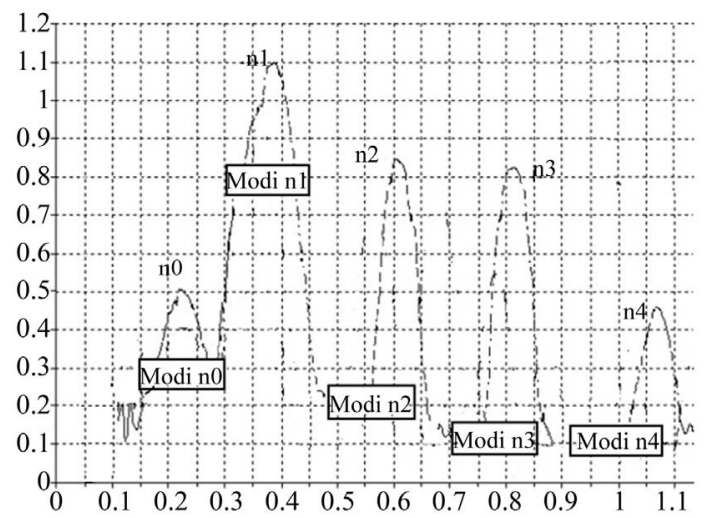

Figure 7. Frequency swep results for different mode.

can be determined by the phase velocity versus the fd to the dispersion curve as it shows Figure 5(b), by calculating the value of phase velocity and known wave mode.

The error for the $\mathrm{n} 1$ is bigger than the others measurements. The other errors are about 1\% - 1.5\%. Greater dispersion of the dispersive curves brings smaller generated error by determination of fd in relation to phase velocity. For $\mathrm{nl}$ mode, the curve is less dispersive at the
Table 1. Measurement results of the thickness with the direct method.

\begin{tabular}{cccc}
\hline $\begin{array}{c}\text { Mode } \\
\text { wave }\end{array}$ & $\begin{array}{c}\text { Frequency } \\
(\mathrm{MHz})\end{array}$ & $\begin{array}{c}\text { Real thickness } \\
(\mathrm{mm})\end{array}$ & $\begin{array}{c}\text { Calculated } \\
\text { thickness }(\mathrm{mm})\end{array}$ \\
\hline $\mathrm{n} 1$ & 0.3310 & 5.89 & 6.28 \\
$\mathrm{n} 2$ & 0.5675 & 5.89 & 6.05 \\
$\mathrm{n} 3$ & 0.8125 & 5.89 & 6.02 \\
$\mathrm{n} 4$ & 1.5225 & 5.89 & 5.97 \\
\hline
\end{tabular}

point of meeting with the line of activation, from where is explained the big error. So in high mode there is greater accuracy in measuring of the thickness.

\section{Conclusions}

Direct method based on achieving peak frequency of shear waves is applied in assessing the thickness of steel non-oxidizing material plate. Algorithm is built for automated drawing of dispersion curves of phase velocity and group speed, the Lamb waves and shear waves. Interpretation of experimental results verifies the below results:

- the generation of the guided waves occurs only at points of dispersion curves and not in the points between them;

- there is distinction between modes of the waves, in terms of improving the reception, sensitivity and power penetration. A good performance of experiments is achieved selecting carefully the phase velocity and frequency;

- n0 mode is useful to see clearly to what kind of wave belongs a peak frequency;

- $\mathrm{n} 1, \mathrm{n} 2$ mode and other types mentioned above can be applied effectively in the evaluation of third parameter (thickness of the planar structures);

- greater dispersion of the dispersive curves brings smaller generated error by determination of $f \cdot d$ in relation to phase velocity.

\section{References}

[1] A. Demma, "Guided Waves: Opportunities and Limitations," AIPND, International Conference, 2009. Available at http://www.ndt.net/article/aipnd2009/files/orig/ 1pdf

[2] J. L. Rose, "A Baseline and Vision of Ultrasonic Guided Wave Inspection Potential," Transactions of the ASME, Journal of Pressure Vessel Technology, Vol. 124, No. 3, pp 273-282, 2002. doi:10.1115/1.1491272

[3] A. Demma, D. Alleyne and B. Pavlakovic, "Uso Delle Onde Guidate per Ispezione di Tuberie Incamiciate o Interrate," AIPND Conference 2005. Available at http://www.ndt.net/article/aipnd2005/files/orig/.pdf

[4] T. Vogt, D. Alleyne and B. Pavlakovic, "Application of 
Guided Wave Technology for Testing," Proceedings of International Conference European NDT, 2009, pp 432438.

[5] T. Kundu, "Ultrasonic Non Destructive Evaluation (Chapter 4)," Cambridge University Press, Cambridge.

[6] J. Rose, "Ultrasonic Waves in Solid Media," Cambridge University Press, Cambridge, 1999.

[7] F. Marques and A. Demma, "Ultrasonic Guided Waves Evaluation of Trials for Pipeline Inspection," 17th World
Conference on Non Destructive Testing, 25-28 October 2008, Shanghai. Available at

http://www.ndt.net/article/wendt2008/papers/96.pdf

[8] Y. Cho, "Guided Wave Monitoring of thickness Variation for Thin Film Materials," Materials Evaluation, Vol. 61, No. 3, 2003, pp. 418-422.

[9] R. B. Thompson, "Physical Principles of Measurements with EMAT Transducers," Physical Acoustics, Vol. 16, 1990, pp. 157-200. 